\title{
Focused Ultrasound Surgery of Breast Cancer: A Computational Study on a Realistic Patient Model
}

\author{
Moslem Sadeghi-Goughari; Soo Jeon; Hyock-Ju Kwon \\ Department of Mechanical and Mechatronics \\ Engineering, University of Waterloo \\ Waterloo, Canada. \\ M8sadegh@uwaterloo.ca, soojeon@uwaterloo.ca, \\ hjkwon@uwaterloo.ca
}

\begin{abstract}
Focused ultrasound surgery (FUS) is a new energybased therapeutic technique which has been recognized as a truly non-invasive surgical method. Since the ultrasonic energy is applied from an external transducer, FUS has the potential to improve both oncologic and cosmetic outcomes for breast cancer treatment. In the present study, we conducted a computational analysis on a realistic patient model to investigate the mechanism and the applicability of FUS in breast cancer treatment. Magnetic Resonance Imaging (MRI) data of a patient with breast tumor were used to provide a clinical model for finite element analysis (FEA) using COMSOL. Results from computational analysis clarify the acoustic and thermal mechanisms of FUS and suggest the way how this technique can be carried out during the breast cancer treatment.
\end{abstract}

Keywords-focused ultrasound surgery; breast cancer; finite element analysis;wave propagation; heat transfer.

\section{INTRODUCTION}

After the cardiovascular and cerebrovascular diseases, cancer is considered as the second leading cause of death in the world, with nearly 8.8 million deaths (15.7\% of total) each year. Breast cancer is the most common cancer among women, with an estimated 1.7 million new cases diagnosed each year in the world [1]. This represents about $25 \%$ of all cancer in women. The Canadian Cancer Society statistics show that about 26,300 new cases of breast cancer have been diagnosed in Canadian women in 2017, and about 5,000 of the cases will be developed to death [2]. The high mortality rate substantiates the importance of early detection and treatment of breast cancers.

Determinant factors in the treatment of a breast tumor are highly dependent on the type, size and location of the tumor [3]. For both benign and malignant cases, surgical techniques such as mastectomy and breast-conserving therapy (BCT) have been considered as the main methods of treatment. Mastectomy, which is the surgical removal of the entire breast, was first introduced by William Halstedt in 1894, and remained as the cornerstone of therapy for about 80 years [4]. Whereas $\mathrm{BCT}$ is achieved just by removing a discrete portion of breast

\author{
Toktam Beheshtian \\ Breast Disease Research Department, Breast Cancer \\ Research Center, Motamed Cancer Institute, ACECR, \\ Tehran, Iran \\ tbeheshtian@gmail.com
}

tissue containing the cancer, and can practically be followed by adjuvant treatment modalities such as chemotherapy and radiotherapy to get more efficient results. Despite the widespread use of surgery as a main treatment for cancer, it is invasive and unable to preserve the structure of the breast postoperatively.

The sensitivity of the human body to the temperature change has caused the development of new therapeutic methods for cancer. Thermal ablation therapy can be achieved by increasing the tissue temperature above a standard level to change the properties of targeted tissue [5]. Some of the energy-based methods used to achieve the thermal ablation of tumors are radiofrequency ablation, laser thermotherapy, and ultrasound therapy. Among them, ultrasound surgery is truly a non-invasive technique, since acoustic waves are emitted by an external transducer [6].

Ultrasound therapies can be split into two major categories, namely "ultrasound hyperthermia" and "high intensity focused ultrasound (HIFU) surgery". During the hyperthermia treatment, the targeted tissue is exposed to the acoustic energy at a low intensity level for a long period of time (10-60 minutes), such that the tumor temperature is evaluated and maintained at $41-45^{\circ} \mathrm{C}$. In contrast, HIFU uses the ultrasonic energy at a high intensity field to increase the temperature of focused area up to $56^{\circ} \mathrm{C}$ with a short ablation time (from 0.1 to 30 seconds) [7].

FUS is a truly non-invasive therapeutic technique which has been recognized for enormous medical applications ranging from cancer treatment to thrombolysis [6]. In principle, FUS allows the local treatment of a tumor by focusing acoustic energies to a targeted area from an extracorporeal source of ultrasound. This process induces tissue heating and corresponding temperature rise in the focal region that would be associated with irreversible biological effects such as necrosis and cell apoptosis.

FUS has been considered as a promising option for the thermal therapy of breast tumors due to its non-invasive mechanism. Since this technique is applied from an external source remotely, it does not cause any significant changes in patients' mammary shape, and has the potential to upgrade both oncologic and cosmetic outcomes for breast cancer 
treatment [8]. In response to the potential benefits of HIFU to patients with breast cancer, many researches have been carried out on the different aspects of HIFU including acoustic-heating characteristics of ultrasound transducer, effects of blood perfusion, and physiological effects of HIFU ablation. During the current decade, the clinical researches on the HIFU treatment of breast cancer were promoted by development of monitoring techniques [8].

In the present paper, we conducted a computational analysis to simulate the wave propagation and heat transfer mechanisms in the FUS of the breast cancer. Finite element model of breast tissue including a tumor was constructed from MRI images of a patient. Using COMSOL software, thermal mechanical results are presented in graphical form to investigate the performance of FUS in the treatment of breast cancer.

\section{MATHERIALS AND METHOD}

\section{A. FUS Mechanism}

Figure 1 shows a schematic drawing of the FUS system to treat breast cancer. When the acoustic waves propagate through the tissue, the medium particles start to vibrate, resulting in alternating cycles of compression and rarefaction pressure inside the tissue [9]. The ultrasound beams then converge into the focal area where the resulting acoustic pressure reaches the highest amplitude. This process leads to temperature rise in the focal region that would be associated with biological ablation and therapeutic results[7].

A single HIFU exposure ablates a small volume of the tumor, so in order to achieve the complete necrosis, the ablating treatment should be applied to the entire volume of tumor [10]. In practice, many of ablated regions must be placed side by side to paint out the entire tumor. The size of each lesion depends on many factors such as the characteristics of transducer and the acoustic properties of targeted tissue, but typically a single insonation covers a cigar shape region with approximate dimensions in the order of 8-15 $\mathrm{mm}$ (along beam axis) $\times 1-3 \mathrm{~mm}$ (transverse direction). The postoperative imaging data show that two weeks following FUS, the periphery of the ablated regions would be replaced by proliferative repair tissue [10].

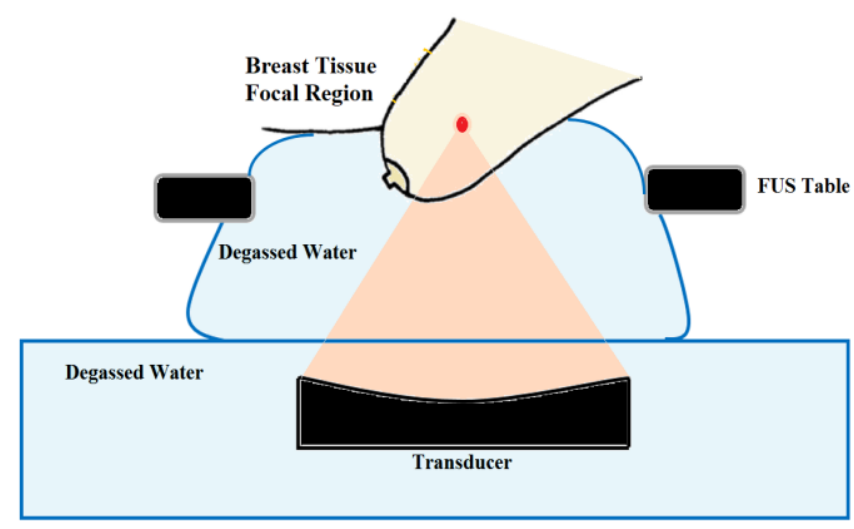

Figure 1. schematic drawing of FUS system used to treat breast cancer

\section{B. Physics and Mathermatics}

The main operation of FUS, is the ablation of a targeted region inside the body by focusing ultrasound beams to that area. [7]. The two predominant factors of FUS, which lead to the therapeutic results, are the conversion of acoustic energy to heat (thermal effect), and the cavitation (mechanical effect). In principle, the role of each effect depends on the dosage parameters, i.e. the intensity at the focal area and the exposure time. If the targeted tissue is exposed to an ultrasound field at a low intensity level for a long period of time, the thermal effect will be the only mechanism of action. However, for applying mechanical effects, a focused energy at a high intensity level for a short time is required [9].

For the mathematical modeling of the focused ultrasound induced heating, the mathematical theories of continuum physics can be used to model the thermal effects of FUS at a relatively low intensity level and before the cavitation threshold [11]. To this end, a set of mathematical equations that describes the wave propagation, absorption and heat transfer mechanisms should be combined together. Accordingly, the ultrasound wave propagation can be characterized with Helmholtz equation, which represents the time independent form of wave equation [12]:

$$
\nabla^{2} P+\left(\frac{\omega}{C_{c}}\right)^{2} P=0
$$

where $P$ is the acoustic pressure, $\omega$ is the angular frequency, and $C_{c}$ is the local speed of sound. To model the heat transfer during FUS, the absorbed acoustic energy can be considered as a heat source for the Pennes bioheat transfer equation [12]:

$$
\rho C_{p} \frac{\partial T}{\partial t}=\nabla \cdot(k \nabla T)-\rho_{b} C_{b} w_{b}\left(T-T_{b}\right)+2 \alpha_{A B S} I,
$$

where $T, \rho, C_{p}$ and $k$ denote the temperature distribution, density, specific heat and thermal conductivity of breast tissue, respectively. $\rho_{b}$ refers to the density of blood, $C_{b}$ is the specific heat of blood, $w_{b}$ is the blood perfusion rate, and $T_{b}$ is the temperature of the blood. $\alpha$ is the acoustic absorption coefficient, and $I$ is the local acoustic intensity which can be determined as a function of the acoustic pressure.

\section{Finite element analysis}

In order to simulate the heating mechanism induced by ultrasound energy and to calculate the acoustic pressure and consequent temperature rise during the FUS process, a FEA using COMSOL software was performed on a realistic patient model. In order to obtain the precise information on the breast tissue and tumor geometries, MRI of a patient with breast tumor was utilized. According to the Helsinki agreement, the whole study was thoroughly explained to the patient. Bilateral MR mammography was performed on a 42 years old lady in the "Erfan imaging center" in Tehran, Iran. The patient was a known case of recently diagnosed breast cancer in the right breast. The images show that there is a $26 \times 17 \mathrm{~mm}$ ill-defined low signal intensity mass in T1W in the right LIQ middle zone. Figure 2 displays the MRI images and consequent 3D solid model of the breast tissue and tumors. 


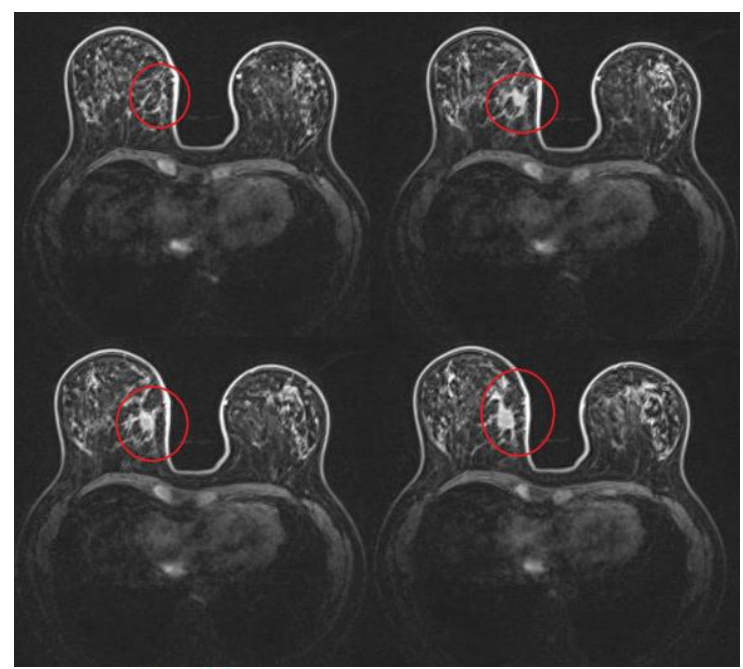

(a)

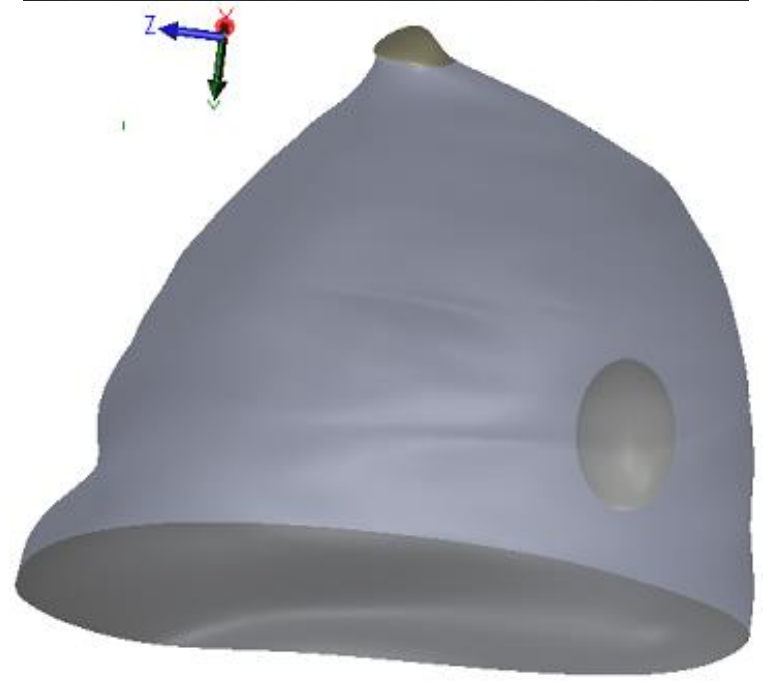

Figure 2. Geometrical features of breast and tumor location: (a) MRI data; (b) Solidworks Model

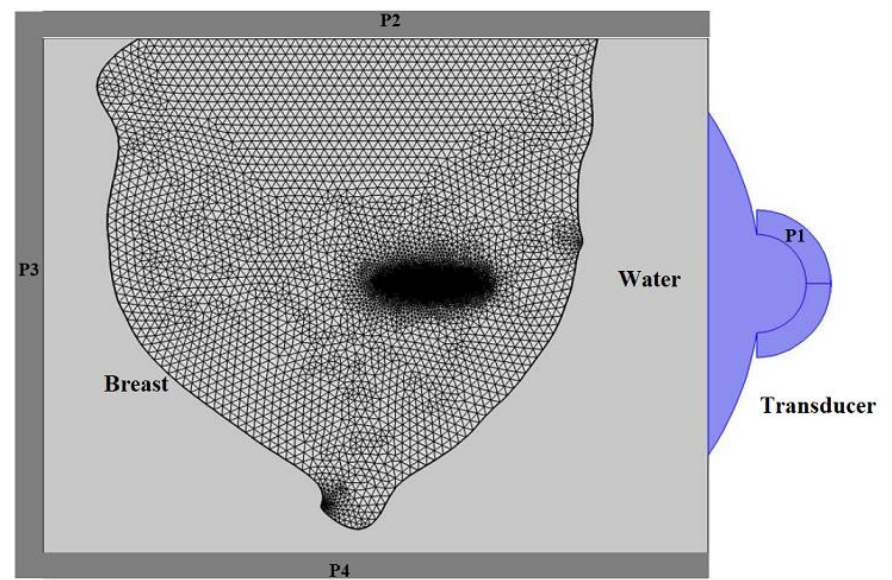

Figure 3. Breast and transducer model for FEA od FUS

A FUS simulation model composed of breast tissue, water and a transducer was developed, as shown in Figure 3. The transducer was bowl-shaped having a focal length of 62.64 $\mathrm{mm}$, an aperture of $35 \mathrm{~mm}$ in radius, and a hole of $10 \mathrm{~mm}$ in radius in the center [9]. A medium of water was considered between the breast tissue and the transducer to act as a coupling material. Four perfectly matched layers (P1-P4) were defined to absorb the outgoing waves. The pressure acoustics simulation was applied to all domains while the thermal analysis was performed only in the breast tissue domain.

The transducer was driven at the frequency, $f$, turned on for 15 seconds, and then turned off to let the breast tissue cool down. For meshing procedure, quadratic (2nd order) elements were used to discretize the temperature in the breast domain and quartic (4th order) elements were used to discretize the acoustic pressure in all domains including breast tissue, water, transducer and PMLs. The acoustic and thermal properties of the water and breast phantom used in the model simulation were derived from Ref [9].

\section{RESULTS AND DISCUSSION}

\section{A. Validation of numerical results}

First, the numerical analysis was developed for a simplified model of water domain to validate the numerical results with those achieved from the experiments [9]. In Figure 4, the normalized pressure profiles in the focal plane are presented, where the results from FEA are compared with those from experiments acquired in water at $30{ }^{\circ} \mathrm{C}$ and at source frequency of $1 \mathrm{MHz}$. The pressure profile from FEA in the present study shows good agreement with the experimental result, particularly in the focal region.

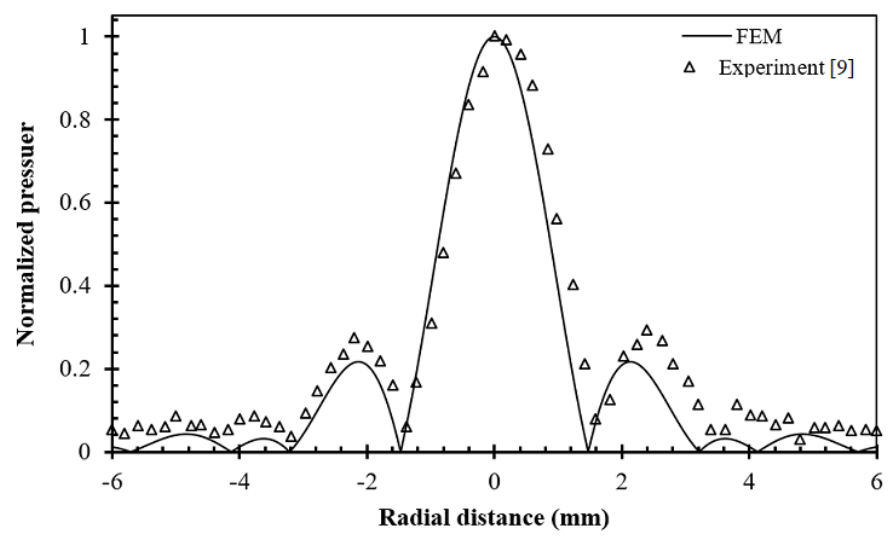

Figure 4. Validation of numerical data with experimental model

\section{B. Acoustic effect}

In this section, the results of wave propagation analysis in the water and the breast domains are presented when the transducer was driven at the frequency of $1 \mathrm{MHz}$. Figure 5 shows the acoustic pressure profile generated by the ultrasound waves propagated through the domains. The figure shows compression and rarefaction regions insides the breast tissue caused by the wave propagation. Here, the waves are focused into the tumor area which lead to a region with high acoustic pressures. A focal region with approximate size of $10 \times 2 \mathrm{~mm}$ is noticeable in the parts of breast which contains cancerous cells. 


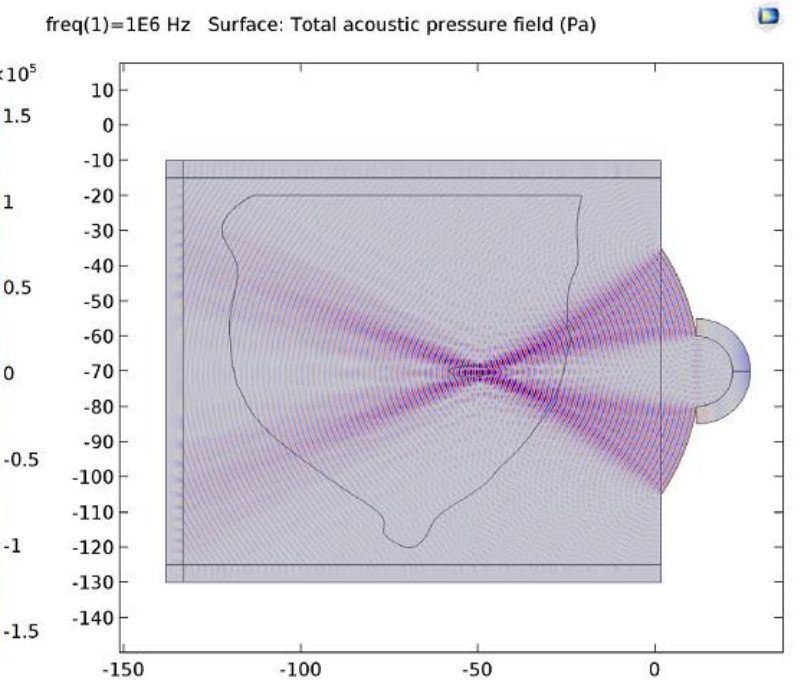

Figure 5. Total acoustic pressure field in breast and water domains.

\section{Thermal effect}

After presenting the acoustic pressure distribution in the breast tissue and water domains, the heat transfer mechanism generated by the absorption of acoustic energy in the breast tissue was determined. Figure 6 shows the temperature distribution in the breast tissue at the different moments of time during the thermal ablation treatment. The figure shows how the temperature rises in the tumor area that needs to be treated. According to the temperature distribution, the maximum temperature rise happened in the cigar-shaped focal region with an approximate length of $10 \mathrm{~mm}$ and a width of $2 \mathrm{~mm}$.
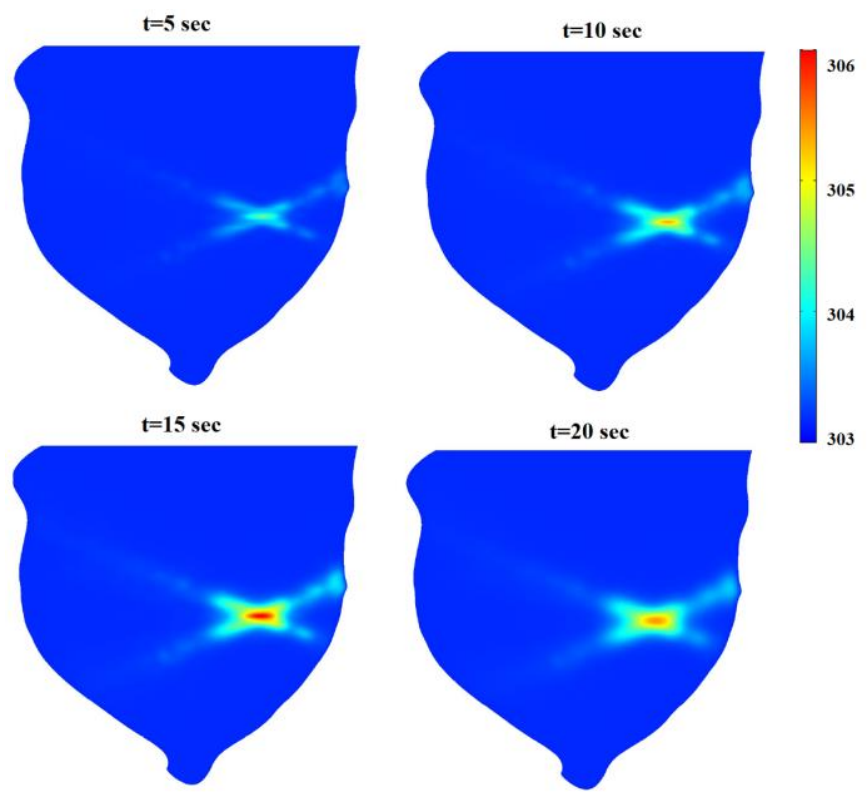

\section{CONCLUSION}

In the present study, a computational study on a realistic patient model was performed to examine the mechanism and the applicability of FUS in the treatment of breast cancer. To this end, the MRI data of a patient with breast tumor were used to mimic the breast tissue and tumor geometry for the finite element analysis of acoustic wave propagation and heat transfer. The results form acoustic and thermal analyses enabled us to understand the mechanism of wave propagation and heat transfer in the breast tissue during the FUS of breast cancer.

\section{ACKNOWLEDGMENT}

This research was supported in part by Natural Sciences and Engineering Research Council of Canada (NSERC), and Government of Ontario under Ontario Trillium Scholarships.

\section{REFERENCES}

[1] R. L. Siegel, K. D. Miller, and A. Jemal, "Cancer statistics, 2016," CA. Cancer J. Clin., vol. 66, no. 1, pp. 7-30, 2016.

[2] www.cancer.ca

[3] G. N. Hortobagyi, "Treatment of breast cancer," N. Engl. J. Med., vol. 339, no. 14, pp. 974-984, 1998.

[4] W. S. Halsted, "I. The results of radical operations for the cure of carcinoma of the breast," Ann. Surg., vol. 46, no. 1, p. 1, 1907.

[5] S. N. Goldberg, G. S. Gazelle, and P. R. Mueller, "Thermal ablation therapy for focal malignancy: a unified approach to underlying principles, techniques, and diagnostic imaging guidance," Am. J. Roentgenol., vol. 174, no. 2, pp. 323-331, 2000.

[6] J. E. Kennedy, "High-intensity focused ultrasound in the treatment of solid tumours," Nat. Rev. Cancer, vol. 5, no. 4, p. 321, 2005.

[7] T. J. Dubinsky, C. Cuevas, M. K. Dighe, O. Kolokythas, and J. H. Hwang, "High-intensity focused ultrasound: current potential and oncologic applications," Am. J. Roentgenol., vol. 190, no. 1, pp. 191-199, 2008.

[8] S. Li and P.-H. Wu, "Magnetic resonance image-guided versus ultrasound-guided high-intensity focused ultrasound in the treatment of breast cancer," Chin. J. Cancer, vol. 32, no. 8, p. 441, 2013.

[9] J. Huang, R. G. Holt, R. O. Cleveland, and R. A. Roy, "Experimental validation of a tractable numerical model for focused ultrasound heating in flow-through tissue phantoms," J. Acoust. Soc. Am., vol. 116, no. 4, pp. 2451-2458, 2004.

[10] J. E. Kennedy et al., "High-intensity focused ultrasound for the treatment of liver tumours," Ultrasonics, vol. 42, no. 1, pp. 931935, 2004.

[11] J. Huang, "Heating in vascular tissue and flow-through tissue phantoms induced by focused ultrasound." Boston University, 2002. modelling by using the finite element method," Phys. Procedia, vol. 63, pp. 127-133, 2015. 